\title{
Indicadores financieros de gestión: análisis e interpretación desde una visión retrospectiva y prospectiva
}

\section{Financial indicators of management: analysis and interpretation from a retrospective and prospective perspective}

Jose Kennedy Ollague Valarezo

Dolores Isabel Ramón Ramón

Carlos Omar Soto Gonzalez

Ernesto Felipe Novillo

Universidad Técnica de Machala, Ecuador

Autor para correspondencia: jollague@utmachala.edu.ec,diramon@utmachala.,edu.ec, csoto@utmachala.edu.ec, enovillo@utmachala.edu.ec

Fecha de recepción: 19 de Julio de 2017 - Fecha de aceptación: 10 de Agosto de 2017

Resumen: Las organizaciones requieren un monitoreo permanente de sus actividades económicas y financieras, las que se pueden realizar mediante la revisión constante de la información plasmada en los estados financieros, aunque resultan insuficientes para brindar al administrador los elementos necesarios para realizar una buena gestión. Para ampliar la visión de la información financiera existe el análisis financiero con su variedad de herramientas como los indicadores financieros, entre los que se encuentran los indicadores de Gestión, Utilidad, Eficiencia, Rotación o Actividad. El objetivo planteado consistió en la propuesta de una visión ampliada de los indicadores de gestión proponiendo dos perspectivas de aplicación, una retrospectiva y otra prospectiva. Para la visión retrospectiva se propuso un diseño de matriz de interpretación de resultados que sirva como elemento de apoyo al momento de tomar decisiones; mientras que para la visión prospectiva se propuso indicadores que permitieron brindar una aproximación a los requerimientos futuros de inversión en activos de una organización en proceso de iniciar operaciones. Se aplicó la matriz de interpretación a un caso ilustrativo útil y resultó adecuada, comprobando su fácil manejo a la hora de integrar la información e interpretar los resultados. Se logró probar la aplicación de las dos visiones y resultó favorable y muy útil recomendando su utilización.

Palabras claves: indicadores de gestión; gestión de activos; indicadores; razones financieras; análisis financiero

Abstract: Organizations require ongoing monitoring of their economic and financial activities, which can be carried out by constantly reviewing the information contained in the financial statements. Unfortunately, financial statements do not provide managers with the necessary elements for good management. Financial analysis with its tools such as financial indicators (indicators of Management, Utility, Efficiency, Rotation or Activity) expand the vision of financial information. The objective of this work was to propose an expanded vision of management indicators, proposing two perspectives of application, a retrospective and a prospective one. For the retrospective view, a matrix of interpretation of results that serves as an element of support at the moment of making decisions was proposed. For the prospective vision, indicators that allowed 
an approximation to the future requirements of investment in assets of an organization in the process of starting to operate were proposed. The interpretation matrix was applied to a useful illustrative case, resulting adequate and proving its easy use in integrating the information and interpreting the results. It was possible to test the application of the two views, showing favorable and useful results, which makes them advisable to use.

Key words: indicators of management; assets management; indicators; financial ratios; financial analysis

\section{Introducción}

Los estados financieros de las organizaciones son elementos muy importantes que permiten observar las condiciones en que se encuentra una empresa. Estos documentos son el resultado del proceso contable que termina con la emisión de informes conocidos como Estados Financieros que informan del estado o situación en que se encuentra una organización. Los Estados financieros solos pueden ofrecer una visión sinóptica de la situación de la organización; sin embargo, esta información resulta insuficiente para emitir una opinión más amplia que permita a la Dirección tomar acciones preventivas o correctivas. Ante esta limitación, se origina la necesidad de realizar un análisis más profundo de esa información financiera, denominado Análisis Financiero, que ofrece varias herramientas de entre las que se destacan los indicadores financieros de los que se destaca un grupo en particular que va a medir la utilización de los recursos de la organización para evaluar su utilización, estos indicadores se van a denominar en la presente investigación indicadores GUERA y son determinantes para una adecuada valoración de la gestión que se realiza en las organizaciones.

El estudio de los denominados indicadores GUERA son claves porque evidencian el nivel de aprovechamiento de las inversiones en activos realizadas en las organizaciones y el impacto en los resultados. En base a lo expuesto se plantea como objetivo una metodología que permita mejorar las aplicaciones de los indicadores GUERA desde dos visiones, una tradicional o retrospectiva y una visión menos convencional, de carácter prospectivo. Se espera que al usar la matriz como elemento de apoyo que forma parte de la propuesta, se logre una mejor y completa interpretación al momento de tomar decisiones.

La investigación logró establecer como adecuada la utilización de una matriz útil para la interpretación y toma de decisiones de los indicadores GUERA de una empresa en marcha; y, se logró demostrar la utilización de estos indicadores reformulados para una visión prospectiva de las organizaciones en formación.

\section{Marco teórico}

El análisis a los Estados Financieros consiste en la aplicación de herramientas y técnicas que relacionan cuentas a fin de obtener información válida para la toma de decisiones. (Bernstein, 1996). Tiene como objetivo evaluar la situación económica y financiera para diagnosticar la situación y perspectivas de un negocio. (Stickney, Weil, Schipper, \& Francis, 2012). Este análisis permite “descubrir" información que no es fácil captar por los usuarios de los Estados financieros, traduciendo a lenguaje comprensible la información financiera. (Villegas, 2002). Estas relaciones entre cuentas y grupos de cuentas de los estados financieros 
denominadas Análisis a los Estados Financieros, abren un amplio abanico de posibilidades para evaluar organizaciones desde varias perspectivas, así tenemos como resultado los denominados ratios, razones, índices, relaciones o indicadores financieros que se constituyen en la base fundamental utilizada para el análisis e interpretación de los estados financieros. (Ibarra, 2006).

De las técnicas utilizadas para realizar análisis financiero, se ha seleccionado los ratios o índices financieros para la presente investigación. Existen cuatro grupos de índices, ratios o razones financieras, que han sido tradicionalmente clasificados por los diferentes autores y que tienen que ver con la liquidez, endeudamiento, gestión y rentabilidad, que además están vinculados con las actividades económicas de las empresas. (Stickney, Weil, Schipper, \& Francis, 2012).

Los indicadores financieros se constituyen en una herramienta indispensable para la adecuada administración financiera de las organizaciones. Son cocientes numéricos que relacionan cuentas o grupos de cuentas de los estados financieros, unos resultan de relacionar cuentas del Estado de Situación Financiera o Balance General, otros de relacionar cuentas del Estado de Resultados y también de relacionar cuentas de ambos estados financieros (Stickney, Weil, Schipper, \& Francis, 2012). Estos indicadores por sí solos no tienen mucho valor, salvo cuando se relacionan unos con otros, cuando se comparan con periodos anteriores y cuando se comparan con empresas del mismo sector (Fontalvo, De la Hoz, \& Vergara, 2012).

Los cuatro grupos de indicadores financieros cubren la totalidad de las cuentas del Estado de Situación Financiera o Balance General y del Estado de Resultados; estos son Indicadores de Liquidez o solvencia; Indicadores de Endeudamiento; Indicadores de Gestión, Utilidad, Eficiencia, Rotación o Actividad; y, los Indicadores de Rentabilidad. De estos cuatro grupos, los Indicadores de Gestión, Utilidad, Eficiencia, Rotación o Actividad, que en este trabajo de investigación se denominarán Indicadores "GUERA" en referencia a su acrónimo basados en las variadas denominaciones otorgadas por distintos autores, relacionan las cuentas del Activo o Patrimonio, del Estado de Situación Financiera o Balance General, con las cuentas del Estado de Resultados.

En la presente investigación es preciso empezar por revisar el enunciado de Activo, debido a que es este rubro el que se va a analizar, para lo cual se toma la definición expuesta por (Guajardo \& Andrade, 2014), quienes consideran "activo" a toda propiedad de una organización capaz de generar beneficios en el futuro, cuyo valor queda definido por el costo de adquisición del bien además de todos los gastos generados por su traslado, instalación, adecuación y puesta en marcha. La cantidad de estos activos varía según la naturaleza de la organización. (Celaya, 2013), agrega, que los activos de las organizaciones pueden ser de diferente tipo y de acuerdo a su naturaleza. Como un buen complemento (Stickney, Weil, Schipper, \& Francis, 2012) presentan la clasificación típica de los Activos en Corrientes y No Corrientes; Activos corrientes son aquellos que se espera su liquidación en el plazo de hasta un año; y, los Activos No Corrientes como aquellos que la empresa retiene por más tiempo y que no se convertirán en efectivo en el corto plazo; es decir, en menos de un año. Con lo que se establece que la diferencia entre el activo circulante y el no circulante depende del tiempo que el activo tarda en convertirse en efectivo (Ochoa \& Saldívar, 2012). 
Como una de las herramientas más utilizadas para una buena gestión está el recurso de Análisis a los Estados Financieros mediante el uso de razones, índices o indicadores financieros, que para (Gitman \& Zutter, 2012), consiste en información contenida en los estados financieros que ofrecen medidas relacionadas al trabajo de la empresa, usando razones o valores relativos que incluyen métodos de cálculo e interpretación para supervisar el desempeño de la empresa.

(Villegas, 2002), define analizar como "romper", "desintegrar", "seccionar" la información de los estados financieros, para posteriormente interpretar, que es el resultado de "integrar" y "sintetizar" los datos descompuestos en el análisis, para alcanzar un entendimiento bajo criterio profesional. Desde esta perspectiva, se propone la utilización tradicional y la innovadora en la forma de operar e interpretar los indicadores GUERA, que según (Van Horne \& Wachowicsz, 2010), son aquellos que permiten medir la gestión basados en la utilización que hace la organización de sus Activos.

Vale iniciar por diferenciar entre lectura e interpretación de los resultados que se obtienen de los indicadores financieros. Defínase la lectura como la expresión literal de un resultado; y, la interpretación, como una profundización al significado de ese resultado, argumentando el impacto que se espera, el posible motivo y las medidas a tomarse, que es justamente la toma de decisiones para remediar o mejorar el curso de la organización.

Los indicadores de Gestión de activos, como los define (Altman, 1968; Amat, O., Leiva,Ll. y Graells, J., 2002), citados por (Bernal-Dominguez \& Amat-Salas, 2012), son considerados como predictores de solvencia y tan importantes que monitorearlos podría evitar el fracaso de las organizaciones y además generar valor. Para (Gitman \& Zutter, 2012) los indicadores de Actividad, como los denomina, miden la velocidad con la que diferentes cuentas se convierten en efectivo, opera en distintas dimensiones, tanto en las cuentas Corrientes como el caso de la administración de inventarios y de las No Corrientes, en donde evalúa la eficiencia con la que se utilizan los activos. Los ingresos por ventas se generarán con mayor eficacia si se combina apropiadamente el efectivo, las cuentas por cobrar, el inventario, plantas, bienes inmuebles y equipo. (Moyer, McGuiran, \& Kretlow, 2005). Estas razones permiten explicar por qué unas empresas con capaces de rotar con mayor rapidez que otras sus activos (Block, Hirt, \& Danielsen, 2013). A continuación se expone cada uno de los indicadores GUERA:

\section{Coeficiente de Rotación de Inventarios (CRI).}

$$
C R I=\frac{\text { Costo de bienes vendidos }}{\text { Inventarios }} \quad \text { (a) } \quad E P I=\frac{\text { Días del año (365) }}{C R I}
$$

El CRI (a) mide la actividad o liquidez que tiene el inventario (Gitman \& Zutter, 2012). Determina además la efectividad con que la organización administra el inventario. Se compara con indicadores pasados, con empresas similares o el promedio de la industria, una mayor rotación define un mejor manejo del inventario (Van Horne \& Wachowicsz, 2010).

Además del CRI, es necesario aplicar otro índice que permite conocer los días de permanencia del inventario en la organización, al que se denominará Edad Promedio de Inventario (EPI) (b) (Gitman \& Zutter, 2012). Indica el tiempo promedio que dura el inventario antes de que se venda (Ross, Westerfield, \& Jaffe, 2012). 


\section{Rotación de Cuentas por Cobrar (RCC).}

$$
R C C=\frac{\text { Ventas a Crédito anuales }}{\text { Cuentas por Cobrar }} \quad \text { (c) } \quad P P C=\frac{\text { Días del año (365) }}{R C C}
$$

La RCC (c) representa las veces que se han generado ventas a plazo y se ha recuperado cartera liberando el efectivo a lo largo del ejercicio (Bernstein, 1996). Como complemento se puede encontrar los días que en promedio se está tomando el cobro de las Cuentas por Cobrar, usando el Periodo Promedio de Cobro (PPC) (d) (Van Horne \& Wachowicsz, 2010):

\section{Rotación de Cuentas por Pagar (RCP).}

$$
R C P=\frac{\text { Costo de Ventas }}{\text { Cuentas por Pagar }} \quad \text { (e) } \quad P P P=\frac{\text { Días del año (365) }}{R C P}
$$

La RCP (e) representa las veces que la organización ha recibido financiamiento de parte de los proveedores y se cancela la deuda. Se complementa con el Periodo Promedio de Pago (PPP) (f) (Gitman \& Zutter, 2012).

Rotación de Activos Fijos (RAF).

$$
R A F=\frac{\text { Ingreso por Ventas }}{\text { Activos Fijos }}
$$

El RAF representa la eficiencia de la organización en generar ingresos utilizando los activos fijos (edificaciones, instalaciones, maquinaria, equipos, etc.); brinda una idea de cuántas veces la organización renueva sus activos fijos en un año, mientras más alto resulta el indicador, supone una utilización más eficiente de los activos fijos (Nava, 2009). Una baja rotación de activos fijos significa que la empresa mantiene capacidad instalada ociosa (Ochoa \& Saldívar, 2012).

\section{Rotación de Activos Totales (RAT).}

$$
R A T=\frac{\text { Ventas }}{\text { Activos Totales }}
$$

El RAT representa la intensidad con la que se utilizan los activos basado en los ingreso por ventas como parte inicial de los futuros beneficios (Bernstein, 1996). Para (Gitman \& Zutter, 2012) indica la eficiencia con la que son utilizados los activos para la generación de ventas. De forma general (Wild, Subramanyam, \& Halsey, 2007) expone que este indicador refleja la productividad relativa de los activos; es decir, de las venta que se derivan de cada dólar invertido en un activo en particular.

\section{Materiales y Métodos}


Los insumos requeridos para desarrollar la propuesta son elementos que forman parte de la información que posee la organización, entre los que están el último estado de Situación Financiera o Balance General, para usar las cuentas del Activo; el Estado de Resultados del último año de donde se obtendrá los valores por Ventas y Costo de los Bienes Vendidos; el Flujo de Caja Neto Proyectado para el inicio de operaciones que proveerá del Pronóstico de ventas con información estimada de las ventas y el costo de venta; $y$, los indicadores financieros GUERA de referencia del giro del negocio al que pertenece la nueva organización para su comparación, análisis, interpretación y toma de decisiones.

Se utilizó el método descriptivo-comparativo, porque describe la manera en cómo se encuentran los indicadores financieros GUERA y compara las formas tradicionales con las formuladas, buscando además con la presente propuesta, ampliar su aplicación en las organizaciones para ser utilizados como elementos dimensionado res para organizaciones que van a iniciar operaciones. Con el propósito de realizar una mejor exposición del tema para la aplicación de los Indicadores GUERA, se ha separado la metodología en dos grupos basados en la perspectiva de aplicación, desde una visión tradicional o retrospectiva y desde una visión innovadora denominada prospectiva.

Desde la perspectiva tradicional o retrospectiva, se utilizarán todos los indicadores GUERA para evaluar el uso que se está haciendo de los activos de una organización en marcha en base a la aplicación de una matriz de evaluación e interpretación, en este primer grupo se clasificará a los indicadores según relacionen las Cuentas Corrientes, con las Ventas y los que relacionan las Cuentas No Corrientes, con las Ventas. En el segundo grupo, desde la perspectiva futura o prospectiva, la información se enfocará partiendo de tres indicadores que resultan clave para ayudar a dimensionar la nueva organización, y son: El CRI, el RAF y el RAT, que se ajustarán para convertirse en UIR, IAF e IAT (ver Figura 1).

\section{CALSIFICACIÓN DE LOS INDICADORES “GUERA"}

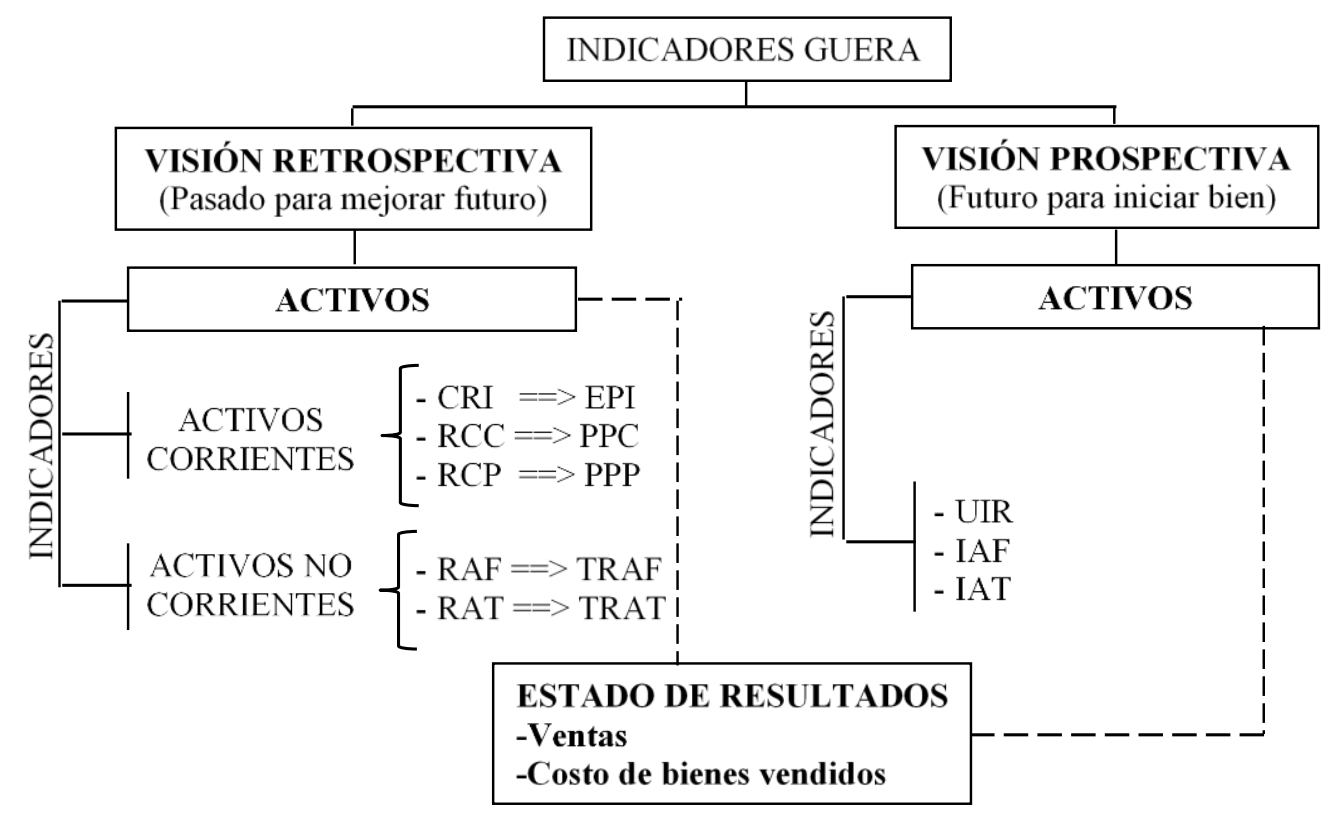


Figura 1. Visión sinóptica de los Indicadores GUERA clasificados por la visión retrospectiva y prospectiva.

Fuente: Elaborado por el autor

\section{Visión retrospectiva}

Así denominada debido a que el análisis financiero se realiza a los estados financieros del periodo fenecido; por tanto, corresponde al pasado, que como ya se expuso, sirve para evaluar la situación financiera de la organización del periodo contable anterior, pero que además, permite tomar medidas correctivas para el siguiente periodo fiscal. Es la forma tradicional de evaluación.

\section{Indicadores GUERA del Activo Corriente o de corto plazo}

El grupo de indicadores GUERA de corto plazo son:

1. Coeficiente de Rotación de Inventarios (CRI) complementado con la Edad Promedio de Inventario (EPI).

2. Rotación de Cuentas por Cobrar (RCC), cuyo complemente es el Periodo Promedio de Cobro (PPC).

3. Rotación de Cuentas por Pagar (RCP), junto con el Periodo Promedio de Pago (PPP).

Este grupo de Indicadores GUERA se debe comparar con los resultados del periodo anterior o periodos anteriores y además con la media de la industria para poder establecer una posición; sea buena, regular o mala con respecto a cada uno de los referentes mencionados y recomendar alguna acción preventiva o correctiva. Para esta actividad se propone una matriz de evaluación que incluye los elementos necesarios para tal valoración (Ver Tabla 1).

\section{Indicadores GUERA del Activo No Corriente o de largo plazo}

El grupo de indicadores GUERA de largo plazo son

Rotación de Activos Fijos (RAF). El RAF representa las veces que la organización recupera en (Ingreso por) Ventas lo invertido en Activos Fijos (Propiedad, Planta y Equipo). Se agrega el tiempo estimado en días que se requieren para alcanzar los valores invertidos en Activos Fijos, para lo cual se propone el indicador denominado Tiempo de Recuperación de Activos Fijos (TRAF), que se obtiene dividendo los días del año con el RAF.

$$
T R A F=\frac{\text { Días del } a \tilde{\mathrm{n}} o(365)}{R A F}
$$

Rotación de Activos Totales (RAT). El RAT representa las veces que la organización recupera en (Ingreso por) Ventas lo invertido en Activos Totales (corrientes y no corrientes). Al igual que los indicadores anteriores, se complementa con el tiempo estimado en días que se requieren para alcanzar los valores invertidos en Activos Totales, para lo cual se propone el 
indicador denominado Tiempo de Recuperación de Activos Totales (TRAT), que se obtiene dividendo los días del año con el RAF.

$$
T R A T=\frac{\text { Días del año (365) }}{R A T}
$$

\section{Matriz de evaluación e interpretación de Indicadores GUERA.}

Los Indicadores financieros requieren de un proceso para evaluar y emitir un criterio mejor sustentado, no es solo leer el resultado, sino que se debe comparar con los valores históricos (al menos un periodo anterior), siendo ideal dos o más periodos para ver la evolución del indicador. Además es indispensable comparar con la media de la industria, puesto que ese será el referente válido para conocer la posición de la organización en el sector al cual pertenece. Para realizar esta actividad, se propone el uso de una matriz que reúne los elementos mencionados y que resultará en un documento de gran apoyo al momento de tomar decisiones, esta es la Matriz de Evaluación e Interpretación de Indicadores GUERA que se presenta a continuación (Tabla 1).

\section{Tabla 1. Matriz de evaluación e interpretación de los Indicadores GUERA correspondientes a la visión retrospectiva del Activo Corriente.}

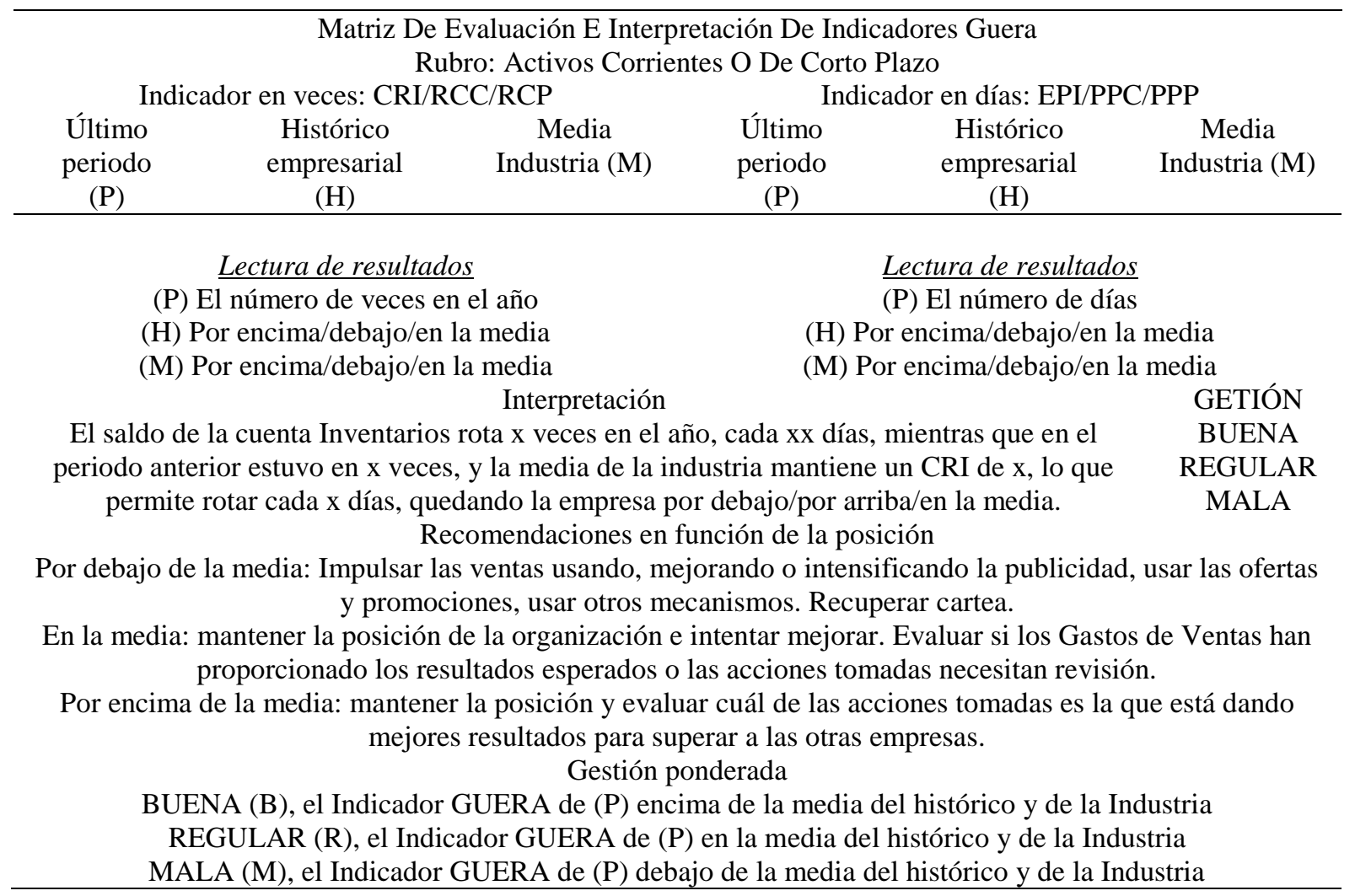

Fuente: Elaborado por el autor. 
Descripción de la matriz. La matriz de evaluación para indicadores GUERA de Corto y largo plazo, que se presenta en la Tabla 1, contiene las casillas de los indicadores de corto plazo expresados en veces y en días. Cada casilla a su vez se divide en tres casilleros en los que se va a registrar el resultado del indicador obtenido por la Empresa en el último periodo $(\mathrm{P})$ o periodo actual de análisis; el resultado histórico $(\mathrm{H})$ obtenido en periodos anteriores; y, el resultado de la media de la industria (M). Luego se procede con la interpretación que se redactará detallando la condición actual $(\mathrm{P})$ con respecto a la anterior $(\mathrm{H})$; a continuación la condición actual $(\mathrm{P})$ con respecto a la media de la industria $(\mathrm{M})$; de acuerdo a estos resultados, se juzgará la Gestión como Buena, Regular o Mala, conforme se recomienda en la matriz. Finalmente se exponen las acciones a tomar dependiendo si los resultados están por debajo, por encima o en la media de la industria. Esta matriz se constituye como elemento de apoyo para la toma de decisiones.

\section{Visión prospectiva}

La segunda parte del estudio consiste en proponer un grupo de indicadores basados en los Indicadores GUERA, que van a permitir dimensionar de manera anticipada la inversión a realizar en Activos Corrientes y No Corrientes de una organización que va a iniciar operaciones en función del Pronóstico de Ventas de su Flujo Neto de Caja (FNC) que para (Sapag, 2011), son los momentos en que se generan los costos y beneficios de un proyecto. En el Flujo de Caja se resume toda la información considerada como relevante en su planeación, como estudio de mercado, estudio técnico entre otros (Andía, 2009) Además de los indicadores GUERA proyectados.

Si una organización realiza el estudio de mercado y los resultados anticipan una determinada cantidad de unidades a vender en el primer periodo, en función de esta información el emprendedor puede definir el espacio aproximado que necesitará para exhibir y mantener el stock de su mercadería al momento de arranque. Ver Figura 2. El Indicador GUERA proyectado para activo corriente es el relacionado a la cantidad de Inventarios o mercancías a requerir.

\section{VISIÓN PROSPECTIVA DE LOS INDICADORES “GUERA” PARA ACTIVOS CORRIENTES}

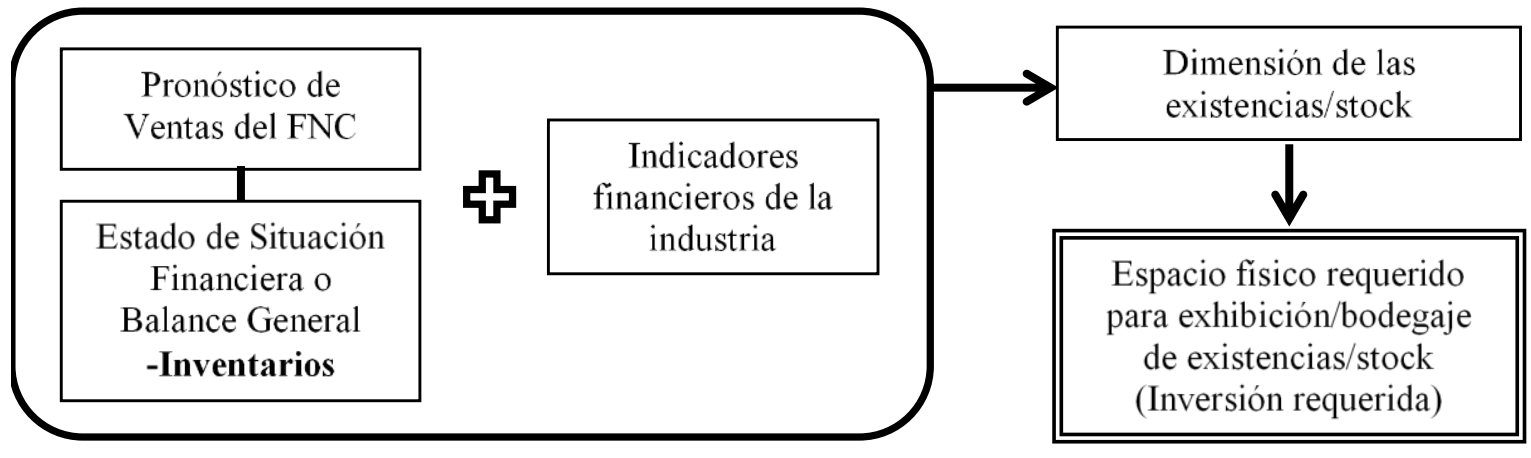

Figura 2. Esquema de la visión prospectiva de los Indicadores GUERA para dimensionar espacios físicos requeridos para exhibición y stock de mercadería.

Fuente: Elaborado por el autor. 
El CRI mide las veces que el saldo de la cuenta Inventarios ha rotado en el año fiscal concluido; es decir, se refiere a lo pasado, lo que sucedió en el periodo contable anterior, como ya se expuso. En el presente estudio el método propone que la relación se efectúe de manera inversa; esto es, realizando un análisis prospectivo usando los indicadores GUERA. Para lograr lo propuesto, se presenta el desarrollo del indicador que se denominará Unidades de Inventario Requerido (UIR).

\section{Unidades de Inventario Requerido.}

La UIR es una aproximación de la cantidad promedio de mercadería que se va a requerir al iniciar y mantener un saldo adecuado en la cuenta Inventarios para mantener rotaciones apegadas a la media de la industria desde el inicio de las operaciones. Además, este indicador dimensiona el espacio físico requerido para su bodegaje o exhibición.

A continuación se desarrollará el proceso conducente a encontrar la relación que formula el indicador.

$$
\begin{gathered}
\text { Saldo Inventario (USD) }=\frac{\text { Costo de bienes vendidos proyectado }}{\text { CRI promedio de la industria }} \\
\text { Unidades de Inventario }(\mathrm{Q})=\frac{\text { Saldo Inventario USD }}{\text { Precio de Costo unitario }} \\
\text { Unides de Inventario }(Q)=\frac{\left(\frac{\text { Costo de bienes vendidos proyectados }}{\text { CRI promedio de la industria } \longleftarrow}\right)}{\text { Precio de Costo unitario } \longleftarrow} \text { - } \\
\text { Unidades de Inventario Requerido } \\
=\frac{\text { Costo de bienes vendidos proyectado } * 1}{\text { CRI Prom Ind } * \text { Precio de Costo unitario }} \\
\text { Unidades de Inventario Requerido } \\
=\frac{\text { Costo de bienes vendidos proyectado }}{\text { CRI Prom Ind } * \text { Precio de Costo unitario }}
\end{gathered}
$$

Con el propósito de aproximarse a la cantidad de inventario (mercadería en stock) recomendable para iniciar los nuevos emprendimientos, como primer paso se comienza por encontrar el saldo tentativo de la cuenta Inventarios (a) del Estado de Situación Financiera o Balance General, para cuyo propósito se requieren dos elementos:

- Costo de Venta proyectado, que se obtiene del Pronóstico de Venta del FNC. 
- Coeficiente de Rotación de Inventario (CRI) del promedio de la industria, que se obtiene de la información financiera de varias empresas relacionadas con la misma actividad en la que se va a emprender en un sector de la economía en particular y así encontrar un CRI promedio de la industria.

Como segundo paso se recomienda encontrar la cantidad aproximada de mercadería requerida para iniciar y mantener el negocio, ver relación (b), para este paso se encuentra la cantidad relacionando el Saldo de Inventario encontrado en (a) con el Precio de Costo Unitario de la mercancía.

El tercer paso será integrar las relaciones (a) y (b) para encontrar las Unidades de Inventario Requerida (c), luego se opera multiplicando extremos con extremos y medios con medios, obteniendo la relación (d), que luego de reducir el factor 1, termina en la relación (e) como la relación financiera creada y propuesta para este propósito denominada Unidades de Inventario Requerida (UIR). Con la UIR obtenemos el requerimiento de las dimensiones de inventario promedio en stock y por tanto el área física que lo va a contener.

\section{Indicadores GUERA para Activos no Corrientes.}

Los indicadores GUERA que establecen alguna medida válida para invertir en infraestructura y dimensionar la inversión de la organización son el IAF (RAF) y IAT (RAT) (ver Figura 3).

\section{VISIÓN PROSPECTIVA DE LOS INDICADORES GUERA PARA ACTIVOS NO CORRIENTES}

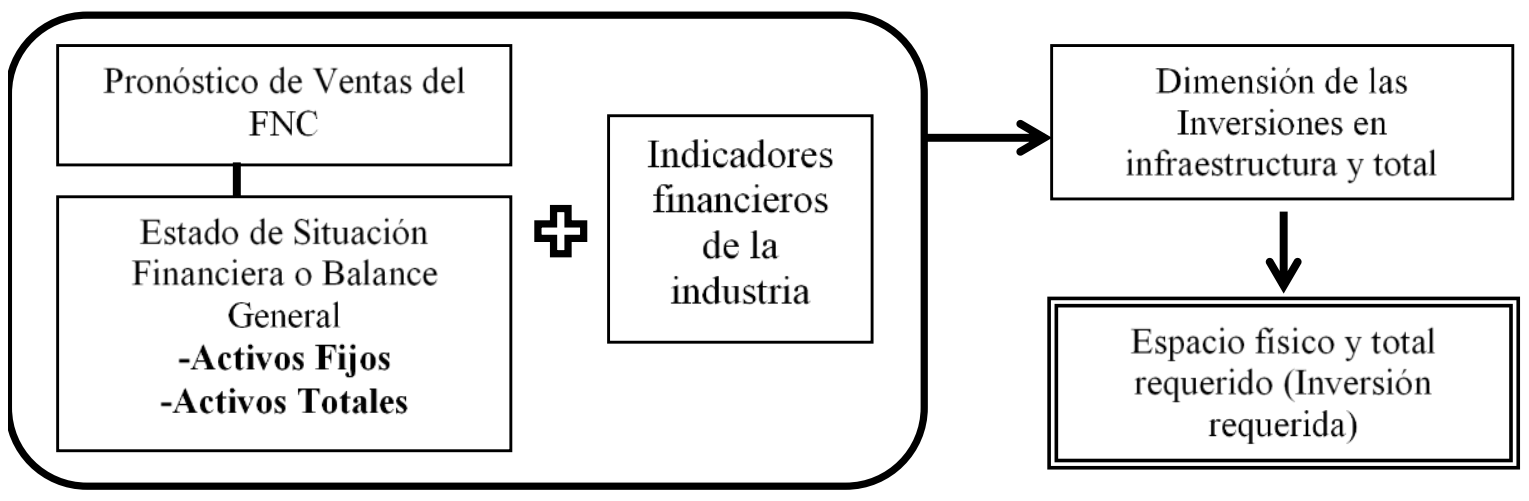

Figura 3. Esquema de la visión prospectiva de los Indicadores GUERA para dimensionar la Inversión en Activos Fijos e inversión Total del nuevo emprendimiento.

Fuente: Elaborado por el autor

\section{Inversión en Activos Fijos (IAF).}

La relación a utilizar para tener un referente aproximado de cuánto invertir en Activos Fijos no resulta muy complicada, puesto que únicamente se toma la información de las Ventas 
proyectadas del Pronóstico de Ventas y el RAF promedio de la industria. La relación resultante es (f):

$$
\text { Inversión en Activos Fijos (IAF) USD }=\frac{\text { Ventas proyectadas }}{R A F \text { Prom Ind }}
$$

\section{Inversión en Activos Totales (IAT).}

Al igual que la relación anterior, la inversión total en Activos, resulta de relacionar las Ventas proyectadas del Pronóstico de Ventas con el RAT promedio de la industria, obtenido al promediar varios RAT de empresas de la misma actividad. Ver relación (g).

$$
\text { Inversión en Activos Totales (IAT) USD }=\frac{\text { Ventas proyectadas }}{\text { RAT Prom Ind }}
$$

Estos indicadores reflejan la dimensión requerida de Activos Totales para la organización que se proyecta crear.

\section{Resultados}

Para comprensión de lo expuesto, se plantea el desarrollo de un caso ilustrativo en el que se aplican las herramientas propuestas. Al proponer dos visiones, se realizan dos casos; uno con la visión retrospectiva y otro con la visión prospectiva.

\section{Visión retrospectiva}

La empresa "Ilustra S.A." dedicada a la venta de equipos de oficina, presenta la siguiente información financiera al 31 de Diciembre de 20XX:

Saldos en las cuentas:

\begin{tabular}{|l|r||}
\hline \hline \multicolumn{2}{|c|}{ DEL BALANCE GENERAL } \\
\hline INVENTARIOS & $25.000,00$ \\
CUENTAS POR COBRAR & $22.000,00$ \\
CUENTAS POR PAGAR & $15.000,00$ \\
ACTIVOS FIJOS (PPYE) & $135.000,00$ \\
ACTIVOS TOTALES & $190.000,00$ \\
\hline
\end{tabular}

S aldos en las cuentas:

\begin{tabular}{||l|r|}
\hline \multicolumn{2}{|c|}{ DEL ESTADO RES ULTADOS } \\
\hline INGRES O POR VENTAS TOTAL & $240.000,00$ \\
VENTAS A CRÉDITO & $160.000,00$ \\
COSTO DE BIENES VENDIDOS & $140.000,00$ \\
\hline
\end{tabular}

\section{Resultados del análisis retrospectivo}

Indicadores Guera De Los

Activos Corrientes O De Corto Plazo

\begin{tabular}{llllll} 
Descripción & Abreviatura & $(\mathrm{P})$ & $(\mathrm{H})$ & $(\mathrm{MI})$ & Lectura \\
Coeficiente de Rotación de Inventarios & CRI & 5,60 & 5,22 & 6,16 & Veces al año \\
Edad Promedio de Inventario & EPI & 65,18 & 69,92 & 59,25 & Días \\
\hline
\end{tabular}


Rotación de Cuentas por Cobrar

Periodo Promedio de Cobro

Rotación de Cuentas por Pagar

Periodo Promedio de Pago

Indicadores Guera De Los

Activos No Corrientes O De Largo Plazo

Rotación de Activos Fijos

Tiempo de Recuperación de Activos

Fijos

Rotación de Activos Totales

Tiempo de Recuperación de Activos

Totales

$\begin{array}{lllll}\text { RCC } & 7,27 & 7,81 & 6,89 & \text { Veces al año } \\ \text { PPC } & 50,19 & 46,73 & 52,98 & \text { Días } \\ & & & & \\ \text { RCP } & 9,33 & 8,49 & 9,30 & \text { Veces al año } \\ \text { PPP } & 39,11 & 42,99 & 39,25 & \text { Días }\end{array}$

$\begin{array}{lllll}\text { RAF } & 1,78 & 1,63 & 1,85 & \text { Veces al año/USD } \\ \text { TRAF } & 205,31 & 223,93 & 197,30 & \text { Días }\end{array}$

$\begin{array}{lllll}\text { RAT } & 1,26 & 1,17 & 1,22 & \text { Veces al año/USD } \\ \text { TRAF } & 288,96 & 311,97 & 299,18 & \text { Días }\end{array}$

Aplicación de la matriz de evaluación e interpretación de los indicadores GUERA.

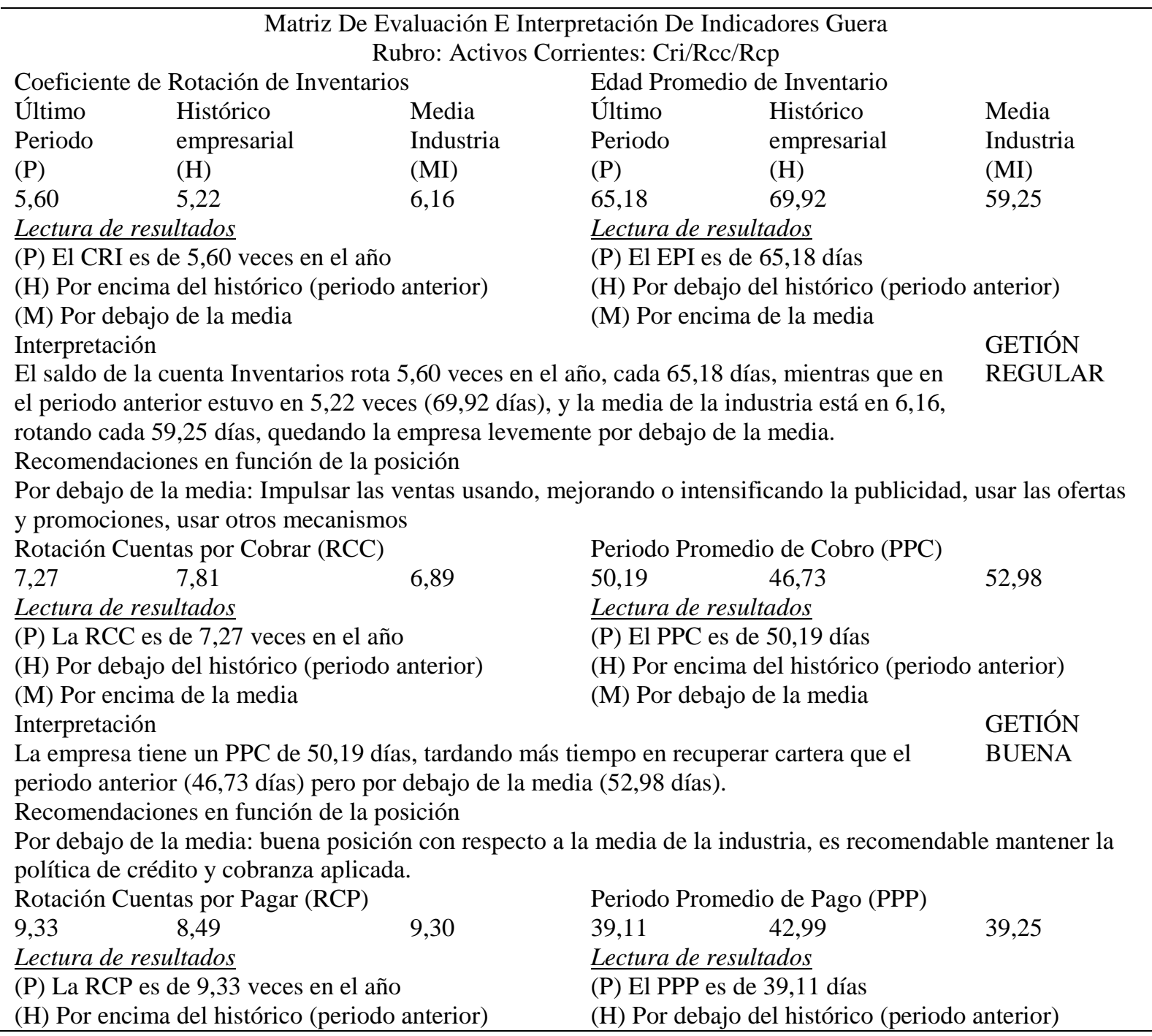




\begin{tabular}{|c|c|}
\hline (M) Por encima de la media & \\
\hline Interpretación & GETIÓN \\
\hline $\begin{array}{l}\text { La empresa tiene un PPP de } 39,11 \text { días, por debajo del histórico, lo que significa que ha } \\
\text { perdido capacidad de negociación con los proveedores en cuanto a plazos, pero se encuentra } \\
\text { por debajo de la media, lo que denota una buena gestión frente al resto de la industria. } \\
\text { Recomendaciones en función de la posición }\end{array}$ & BUENA \\
\hline $\begin{array}{l}\text { Por debajo de la media: buena posición con respecto a la media de la industria, es recomend } \\
\text { capacidad de negociación de los plazos con los proveedores pero tratar de recuperar los valo }\end{array}$ & $\begin{array}{l}\text { le mantener la } \\
\text { históricos. }\end{array}$ \\
\hline
\end{tabular}

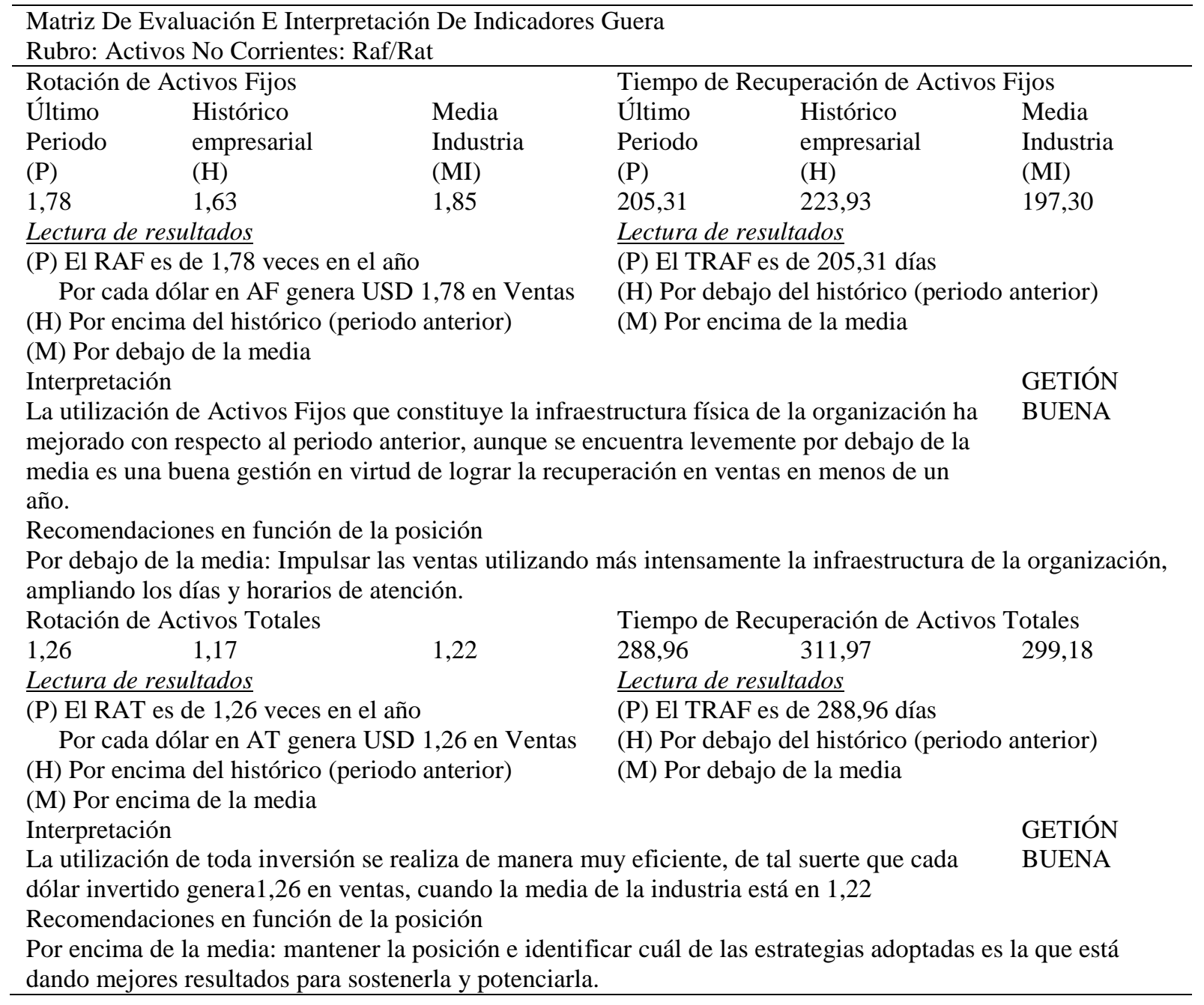

\section{Visión prospectiva}

El segundo enfoque propuesto para los Indicadores GUERA es el prospectivo; es decir, el que permitirá utilizar información futura para usar como referencia al inicio de las operaciones. A continuación se aplica a un caso práctico.

Se proyecta iniciar un emprendimiento para la venta de refrigerios, los que según el estudio realizado por el especialista del proyecto, se van a adquirir a USD 1,20 y podrán ser 
vendidos a USD 2,00 la unidad, con un margen bruto de USD 0,80 por unidad vendida. El estudio de mercado dio como resultado la venta potencial de 10.000 unidades para el momento de arranque del emprendimiento o del próximo periodo.

La investigación realizada para obtener los indicadores GUERA, encontró lo siguiente:

Indicadores GUERA obtenidos del promedio de la industria.

\begin{tabular}{|c|c|}
\hline \multicolumn{2}{|c|}{ INDICADORES PROMEDIO INDUSTRIA } \\
\hline CRI PROM IND $=$ & 9 \\
\hline RAF PROM IND $=$ & 2 \\
\hline RAT PROM IND $=$ & 1,7647 \\
\hline
\end{tabular}

Con la información recabada y pertinente se puede obtener los indicadores propuestos conforme se expone a continuación:

\begin{tabular}{lccc}
\multicolumn{1}{c}{ DESCRIPCIÓN } & AÑO 1 & AÑO 2 & AÑO ... \\
\hline Refrigerios & $10.000,00$ & $10.125,00$ & $\ldots$ \\
Precio Venta Unitario & 2,00 & 2,00 & $\ldots$ \\
\hline Subtotal Ingreso por ventas & $20.000,00$ & $20.250,00$ &
\end{tabular}

\begin{tabular}{l|c|cc}
\multicolumn{1}{l|}{\begin{tabular}{l} 
PRONÓSTICO DE VENTA \\
\multicolumn{1}{c|}{ DESCRIPCIÓN }
\end{tabular}} & AÑ̃ 1 & AÑo 2 & AÑo ... \\
\hline Ingreso por Ventas & $20.000,00$ & $20.250,00$ & $\ldots$ \\
Costo de Venta & $12.000,00$ & $12.150,00$ & $\ldots$ \\
\hline \multicolumn{1}{c|}{ UTILIDAD BRUTA } & $\mathbf{8 . 0 0 0 , 0 0}$ & $\mathbf{8 . 1 0 0 , 0 0}$ & $\ldots$
\end{tabular}

El pronóstico de ventas que se presenta en la Tabla T1, es tomado del FNC del proyecto de inversión que resume la información necesaria y suficiente como para comprensión de la propuesta con fines ilustrativos. Se puede observar los datos a utilizar en el análisis prospectivo: la cantidad de unidades estimada, el precio unitario para venta y el costo del producto a vender, el ingreso por venta total y el costo de venta total estimado para el arranque del emprendimiento.

La tabla T2 presenta los indicadores GUERA obtenidos de la media de la industria a la que la nueva organización se dedicará. Con los materiales necesarios ya se puede proceder con el análisis prospectivo bajo la metodología propuesta.

\section{Unidades de Inventario Requeridas (UIR).}

Se procede a obtener la cantidad en unidades de mercadería (Inventario) que van a servir de referencia para la inversión en Activos corrientes de la nueva organización. Aplicando la fórmula se obtiene: 
$\checkmark$ Unidades de Inventario Req $(\mathrm{Q})=12.000 /(9 * 1,20)$

$\checkmark$ Unidades de Inventario Requerido $=1.111,11=1.111$ unidades (refrigerios)

$\checkmark$ Lectura: 1.111 refrigerios es el inventario requerido.

Interpretación: La empresa va a requerir espacio físico para aproximadamente 1.111 unidades de "refrigerios" para iniciar el negocio con un stock adecuado, ese stock es referencial y será el que se espera rote por unas nueves veces en promedio durante el año.

El UIR aporta con una estimación del espacio físico requerido en función del tipo de mercancía, lo cual resulta muy útil a la hora de invertir en el local en donde se establecerá la empresa, pero igual ayuda a establecer el valor económico del Inventario al multiplicar el UIR por el precio unitario, que en este caso sería USD 1.333,33, en promedio.

\section{Inversión en Activos Fijos Requeridos (IAFR).}

Este indicador busca dimensionar la inversión necesaria a realizar en Activos Fijos para no resultar sobrecargado de infraestructura, para el presente caso, el resultado es de USD 10.000,00 requerido como máxima inversión en Activos Fijos.

$$
\text { Inversión en Activos Fijos USD }=\frac{20.000,00}{2}=10.000,00
$$

\section{Inversión en Activos Totales Requeridos (IATR).}

En el caso de los Activos Totales, lo conveniente es no realizar inversiones superiores a USD 11.333,37.

$$
\text { Inversión en Activos Totales USD }=\frac{20.000}{1,7647}
$$

$$
\text { Inversión en Activos Totales = 11.333,37 dólares }
$$

Aplicando esta metodología se podrá tener una idea aproximada de cuánto se va a necesitar para montar y poner en marcha la organización afectando lo menos posible la eficiencia ayudados por la aplicación de los indicadores GUERA desde la visión prospectiva, debido a que, como ya se ha mencionado, estos indicadores son determinantes para el éxito o fracaso de las organizaciones.

\section{Discusión}

Se puede deducir que la utilización de los Indicadores de Gestión, Eficiencia, Utilidad, Rotación o Actividad (GUERA), son claves para determinar la cantidad de recursos apropiados que deben tener las organizaciones desde dos perspectivas; la primera, como indicadores que 
miden la gestión de los activos de una empresa en marcha como recurso definitivo para obtener buenos resultados; $y$, la segunda, como unidad de medida prospectiva para ayudar a dimensionar apropiadamente a la organización que va a iniciar operaciones, en cuanto a la cantidad de inventario y el espacio requerido, el saldo en valor económico, las inversiones en activos fijos y activos totales a realizar.

Desde la perspectiva del análisis de una empresa en marcha, el presente enfoque coincide mayoritariamente con lo propuesto por (Nava, 2009), que interpreta los resultados de los indicadores en función del máximo aprovechamiento de los recursos de la organización. Igualmente con la mayoría autores que exponen el análisis de los indicadores GUERA desde una visión retrospectiva quizá muy simple, enfocándose únicamente en algunas relaciones y una lectura de resultados sin elementos que fortalezcan una mejor interpretación que ayude al administrador a evaluar el uso conveniente que se ha hecho de los activos de la empresa y constatar si se aprovecha bien de la infraestructura, o poder tomar medidas correctivas para mejorar la presente y futura gestión.

Además se ha demostrado que los indicadores GUERA tienen una aplicación mucho más amplia de lo tradicionalmente utilizado puesto que sirven para dimensionar futuras organizaciones apegando las inversiones a cifras de la industria que en muchas ocasiones podrían estar muy alejadas en menor o mayor proporción a las previstas sin esta aplicación. En ambos casos, ya sea en una menor o mayor cantidad de activos a la necesaria, va a repercutir en el éxito o fracaso de la organización. Una deficiente cantidad de activos reflejará un problema de insatisfacción a los potenciales clientes debido a la falta de cumplimiento en atender pedidos y también en la cantidad de inventario a considerar para mantener en stock, mientras que un exceso de activos, repercutirá negativamente en la eficiencia del uso de éstos, al reflejar una sobrecarga de activos inactivos, rompiendo la esencia de la definición que se ofreciera al inicio de este documento de que los activos deben ser generadores de riqueza.

Vale mencionar que existen herramientas financieras para dimensionar apropiadamente el inventario como es la Cantidad Óptima de Pedido (QEP), que intenta dimensionar justamente cuál es la cantidad óptima a pedir cada cierto tiempo para disminuir al máximo el costo de gestión de inventarios, pero que no obsta en la utilización de los indicadores financieros GUERA como apoyo y fortalecimiento a esta y cualquier otra herramienta.

Además es necesario destacar que en trabajos como el realizado a empresarios de las PYMES por (González \& Bermúdez, 2008), que dan mayor énfasis a los indicadores de rentabilidad, aunque identifican otros indicadores de carácter no financiero con niveles de importancia en el proceso de toma de decisiones, denota claramente el desconocimiento de la importancia de los indicadores GUERA en la buena gestión de las organizaciones. Una empresa no podría alcanzar significativos niveles de rentabilidad sino gestiona adecuadamente sus Activos (patrimonio), se debe tener presente además que la rentabilidad es solo el resultado de una gestión, más la gestión misma y toma de decisiones está en la utilización eficiente de los recursos de la organización.

De la misma manera vale destacar definiciones de análisis financiero como la expuesta por (Morelos, Fontalvo, \& De la Hoz, 2012) quienes afirman que el análisis financiero se hace en 
base a información existente por lo que no se enfatiza en el futuro, aunque aclara que lo evaluado en el pasado sirve para proyecciones en el futuro; sin embargo, no deja de ser una visión tradicional sobre la aplicación de los Indicadores financieros, a diferencia de la presente visión en donde se utiliza estos elementos del análisis financiero con aplicaciones más amplias.

\section{Conclusiones}

Se realizó una revisión de algunos autores para determinar el uso y aplicación a la Gestión de activos, mediante indicadores financieros que, en el presente estudio, se los ha denominado indicadores GUERA.

Se espera que los indicadores GUERA propuestos desde su visión retrospectiva y prospectiva, sean apropiados para ser aplicados en las empresas en marcha así como en los nuevos emprendimientos, puesto que en muchas ocasiones una falta de análisis adecuado podría estar generando una sobrecarga de activos o un mal aprovechamiento de éstos.

El uso de la matriz de interpretación de los Indicadores GUERA se constituye en un instrumento de apoyo para la toma de decisiones, al reunir en un solo documento los elementos necesarios que se deben considerar en el momento de interpretar los resultados y emitir las recomendaciones pertinentes.

Se propone la aplicación del método como un elemento adicional a los existentes para abundar en opciones que permitan lograr el éxito de las organizaciones y evitar en lo posible su debilitamiento y más aún su fracaso. 


\section{Bibliografía}

Andía, W. (2009). Proyectos de inversión (Segunda ed.). Lima: El saber.

Bernal-Dominguez, D., \& Amat-Salas, O. (mayo-agosto de 2012). Anuario de ratios financieros sectoriales en México para análisis. Ra Ximhai, 8(2), 271-286.

Bernstein, L. (1996). Análisis de Estados Financieros. Barcelona: Irwin.

Block, S., Hirt, G., \& Danielsen, B. (2013). Fundamentos de Administración financiera (Decimocuarta ed.). México: McGraw-Hill.

Celaya, R. (2013). Contabilidad básica. Un enfoque basado en competencias. México D.F.: Cengage learning.

Fontalvo, T., De la Hoz, E., \& Vergara, J. (24 de agosto de 2012). Aplicación de análisis discriminate para evaluar el mejoramiento de los indicadores financieros en las empresas del sector alimento de Barranquilla-Colombia. Ingeniare. Revista chilena de ingenieros, 20(3), 320-330.

Gitman, L., \& Zutter, C. (2012). Principios de Administración financiera (Decimosegunda ed.). México: Pearson Educación.

González, P., \& Bermúdez, T. (junio de 2008). Una aproximación al modelo de toma de decisiones usado por los gerentes de la micro, pequeñas y medianas empresas ubicadas en Cali, Colombia desde un enfoque de modelos de decisión e indicadores financieros y no financieros. Contaduría Universidad de Antioquia (52), 131-154.

Guajardo, G., \& Andrade, N. (2014). Contabilidad financiera (Sexta ed.). México D. F.: McGrawHill.

Herrera, C., \& Montoya, L. (15 de marzo de 2013). El Emprendedor: una aproximación a su definición y caracterización. Punto de vista, IV (7), 7-30.

Ibarra, A. (Septiembre de 2006). Una perspectiva sobre la evolución en la utilización de las razones financieras o ratios. Pensamiento y gestión (21).

Morelos, J., Fontalvo, T., \& De la Hoz, E. (enero-junio de 2012). Análisis de los indicadores financieros en las sociedades portuarias de Colombia. Entramado, 8(1), 14-26.

Moyer, C., McGuiran, J., \& Kretlow, W. (2005). Administración financiera contemporánea (novena ed.). Cengage learning.

Nava, M. (20 de 0ctubre de 2009). Análisis financiero: una herramienta clave para una gestión financiera eficiente. Revista Venezolana de Gerencia (RVG), año 14(48), 606-628. 
Ochoa, G., \& Saldívar, R. (2012). Administración financiera correlacionada con las NIF (Tercera ed.). México: McGraw-Hill.

Ross, S., Westerfield, R., \& Jaffe, J. (2012). Finanzas corporativas (Novena ed.). México.

Sapag, N. (2011). Proyectos de inversión. Formulación y evaluación (Segunda ed.). Santiago de Chile: Pearson Education.

Stickney, C., Weil, R., Schipper, K., \& Francis, J. (2012). Contabilidad Financiera (Primera ed.). Buenos Aires: Cengage Learning.

Van Horne, J., \& Wachowicsz, J. (2010). Fundamentos de Administración Financiera (Décimotercera ed.). México: Pearson educación.

Villegas, E. (enero-junio de 2002). Análisis financiero en los agronegocios. Revista mexicana de agronegocios, VI (10).

Wild, J., Subramanyam, K., \& Halsey, R. (2007). Análisis de Estados Financieros (Novena ed.). México. 